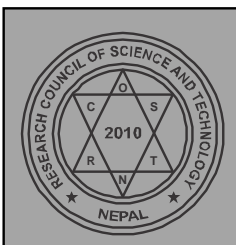

\title{
BIBECHANA
}

A Multidisciplinary Journal of Science, Technology and Mathematics

ISSN 2091-0762 (online)

Journal homepage: http://nepjol.info/index.php/BIBECHANA

\section{Weak interactions-Strong effects: Key role of hydrophobic interaction in the formation of stimuli-responsive viscoelastic gel of worm-like micelles}

\author{
Swapan K. Saha* \\ Department of Chemistry, University of North Bengal, Darjeeling, 734 013, India \\ E-mail: ssahanbu@hotmail.com
}

Accepted for publication: February 02, 2014

\begin{abstract}
Among all the weak interactions, which are operative in the domain of chemistry, hydrophobic effect is one that leads to new structural motifs like molecular self-assemblies viz., micelles, vesicles etc. Worm-like micelles are polymer like aggregates which have been formed in aqueous surfactant solutions in the presence of additives. These are relatively new materials with exciting material properties. These stimuli-responsive viscoelastic materials are interesting from both fundamental understanding as well as application points of view. The physico-chemical properties of worm-like micelles are studied and the morphological transitions to vesicle as functions of various parameters have been investigated.
\end{abstract}

(C) 2014 RCOST: All rights reserved.

Keywords: Hydrophobic effect; Vesicles; Worm-like micelles; Viscoelastic materials.

\section{Introduction}

From the days of alchemists, the primary goal of chemistry has been to understand the behavior of molecules and their construction from constituent atoms. In recent years chemists have extended their goal beyond the atomic and molecular chemistry into the realm of supramolecular chemistry controlled by weak interactions. In chemistry, weak interactions are the intermolecular forces mainly governed by (i) vander Waals interaction $(0.5-2 \mathrm{kcal} / \mathrm{mol})$ (ii) Hydrophobic interactions $(1-2 \mathrm{kcal} / \mathrm{mole})$ (iii) $\pi-\pi$ interaction (2-3 kcal/mole) and (iv) Hydrogen bonding (12-16 kcal/mole). Among all the weak interactions, which are operative in the domain of chemistry, hydrophobic effect is one that leads to new structural motifs like molecular self-assemblies viz., micelles, vesicles etc. in aqueous media.

The unfavorable contact between water and the apolar part of surfactant molecules lead to their congregation into well organized entities, viz., micelles, vesicles, fibers, discs and tubes [1-3]. Although micelles are usually spherical in shape, under certain conditions, e.g., concentration, salinity or in the presence of hydrophobic counter ions, etc., they may undergo uniaxial growth. This subsequently results in the formation of significantly long $(100-500 \mu \mathrm{m})$ yet highly flexible aggregates referred to as

\footnotetext{
Prof. Swapan K. Saha,

Keynote Speaker

International Conference on Emerging Trends in Science and Technology

(March 22-23, 2014) Biratnagar, Nepal
} 
"worm-like micelles (WLM)" [4-6]. The research of WLM has drawn considerable interest as its rheology is very challenging due to the presence of multiple pertinent length scales and stress relaxation mechanisms. This relatively new material has many applications including that of fractured fluids in oil fields, efficient drag reducing agent in hydrodynamic engineering and home care, personal care and cosmetic products [7-8]. Viscoelastic WLMs are formed in various surfactant systems in the presence of different additives [9-12]. Most extensively studied system is the cetyltrimethylammonium bromide (CTAB) micelles in presence of hydrotrope, sodium salicylate (SS). Unlike simple halides, salicylate promotes sphere to wormlike micellar transition at very low concentrations, viz., near the normal critical micelle concentration $(\mathrm{cmc}, \sim 1 \mathrm{mM})$ of CTAB. The flexible and elongated wormlike micelles under dilute conditions show complex and unusual rheological phenomena, which include strong viscoelasticity and shear-induced structure (SIS) formation. It is particularly interesting that, while a wide variety of wormlike ionic micellar solutions display identical rheological responses, a common element in most of these systems was the presence of salt anions such as SS.

In view of the importance of an efficient WLM promoter, which might be effective for various applications at low surfactant concentrations and in the presence of metal ion impurities, organic $\pi$ conjugated molecules with H-bonding functionality, viz., naphthols are highly promising [13-16]. Microstructural transitions of micellar aggregates, especially the nature of transition from ordinary micelles to long worm-like giant micelles and the vesicles, mediated by organic $\pi$-electron systems are of fundamental scientific interest and have been reported in several papers only recently [17-18]. Moreover, synthetic vesicular systems are interesting from a number of standpoints, not the least being their structural similarity with the constituent of the biomembrane, viz., phospholipid. They offer a convenient way to probe interactions involving membrane systems. Vesicle aggregation or adhesion is the primary step for the fusion of the vesicles in membrane. Therefore, the elucidation of the molecular mechanism of vesicular aggregation would greatly contribute to a better understanding of these biological phenomena.

Further, it may be anticipated that a simple and effective route to design a $\mathrm{pH}$ responsive microstructure could well be based on the neutral naphthol dopants, which form salts only at high $\mathrm{pH}$ (pKa 9.2). As a function of $\mathrm{pH}$, ionization of the $\mathrm{OH}$ group of naphthol molecules may switch the onset of charge screening, paving the way to effect further morphological transitions (viz., vesicle formation). One of the objectives of the present work is, therefore, to design a simple effective route of $\mathrm{pH}$-responsive morphological transition for the aqueous molecular aggregates of single chain cationic surfactant, viz., CTAB from micelles to long wormlike micelle to unilamelar vesicles.

\section{Historical Overview}

Since the pioneering work of Prof. H. Hoffmann [9] and his group of Bayreut University, Germany on the unusual rheological property of WLM in the year 1988, quite a large number of physical chemists, physicists and chemical engineers became interested in this fascinating area and have contributed immensely to the advancement of WLM research. Among others this list includes Prof. S. Candau and M. Cates [19-20] of Louis Pasteur University, France; Prof. H.T. Davis [21], University of Minnesota, U.S.A.; Prof. D.J. Pine [22] and Prof. S.L. Keller [23], both from the University of California, U.S.A.; Prof. E.W. Kaler [24], University of Delware, U.S.A. and Prof. T. Imae [25,26] of Nagoya University of Japan. The coevolution of strong theoretical framework alongside application of a range of sophisticated experimental tools engendered rapid advancement in the study of WLM. Presently the main focus of research in this field has been shifted to the development of new surfactant systems and new protocol by which WLM formation could be triggered. Prof. S.R. Raghavan [27] and his group of University of Maryland have developed a series of new shear, temperature and light responsive WLM systems, which are of high technological interests. A mixture of CTAB and trans orthomethoxy cinnamic acid (OMCA) (photo-rheological fluid) form long wormlike micelles leading to strong viscoelasticity under the 
influence of UV light. This trans OMCA is converted to cis isomer of OMCA, which interacts only weakly with $\mathrm{CTAB}$ and eventually viscoelasticity disappears. Prof. Raghavan's group has also studied WLM to vesicle transition as influenced by heat. Prof. A. Bose [28] of University of Rhode Island and Prof. V.T. John [28] of Tulane University, USA has investigated surfactant solubilization and direct encapsulation of interfacially active phenols in mesoporous silicas. Template synthesis of mesoscopic tube silicates using aqueous mixtures of naphthalene diol and ammonium surfactant has been carried out by Prof. M. Isayama [29] of Kyushu University, Japan. Dr. R. G. Shrestha [30] and her groups, also from Japan, are involved in extensive research on WLM with the aid of SAXS, rheometer, cryo-TEM etc. on some novel and interesting systems including poly (oxyethylene) cholesteryl ether. In China, the researchers in the field of WLM include Dr. G-Z. Li [31] of Shardong University, Dr. S. Liu [32] of Science and Technology University and Prof. J. Huang [33] of Peking University. Dr. L. Zheng [34] of Key Laboratory of Colloid and Interface Chemistry has been engaged in WLM research involving surface active ionic liquids (SAIL). In view of the fascinating rheology and technological applications of WLM, a tunable and efficient method of preparation of WLM is required. In spite of remarkable interests among the scientists on this exciting area of research, there is a major gap in the method of preparation and triggering of the WLM formation from a wide range of surfactants by metal ion inert neutral promoters (hydroxyl aromatic compounds). Unless this limitation is addressed properly a complete understanding of the phenomenon, its application and control may prove to be far from complete success.

\section{Results and Discussion}

Despite high promise shown by neutral hydrotropes like naphthols in triggering the formation of WLM from cationic surfactants, surprisingly these hydroxyl aromatic compounds have been used only scarcely. Aqueous CTAB (2-10 mM) and 1- or 2-naphthol (2-10 $\mathrm{mM}$ in 2-5\% methanol, naphthols being sparingly soluble in water) solutions show viscosities similar to those of water. But as soon as these solutions are mixed together at room temperature, a thick gel-type fluid with high viscoelasticity is developed.

From the initial visual observation it was found that the viscosity of the gel was very much dependent on the concentration of CTAB and the additives. Therefore, we first determined the CTAB: dopant mole ratio at which the gel shows maximum viscosity [14]. Much like the CTAB-NaSal system, it was found that CTAB-naphthols also display maximum viscoelasticity at a 1:1 molar ratio of surfactant and the promoter (Fig. 1). On the other hand the effect of the dihydroxynaphthalenes (2,3-DHN and 2,7-DHN) on micelles of $\mathrm{CTAB}$ are completely different. Equimolar mixtures of only CTAB and 2,3-DHN gives a highly viscous gel in aqueous solutions but 2,7-DHN/CTAB does not. Therefore, further attempts were not made to study the viscosity of 2,7-DHN/CTAB system [15]. The argument that an excess or deficiency of charge on the micelles due to adsorption of hydrotrope anions (e.g., NaSal) would shorten the micellar life time and size is not apparently true for the present system because under the present experimental condition of solution $\mathrm{pH}(\sim 6.5)$, the naphthols and the dihydroxynaphthalenes are mostly protonated, i.e., uncharged ( $\mathrm{pKa}$ 's > 9.0) [16]. Therefore, it seems apparent that the symmetrical distribution of surfactant and the promoter molecules leading to highly compact spherical micelles facilitates an optimum surface curvature to attain in presence of $\mathrm{H}$ bonding (discussed later), and this results in the sphere to rod transition easily. For further experiments, dopant to surfactant ratio was chosen to produce strongest viscoelasticity, i.e., 1:1 mole ratio.

Further, the rheological responses for a viscoelastic system (viz., an aqueous CTAB-1-naphthol system as a function of concentration $(1: 1 \mathrm{~mol}$ ratio $)$ at $\left.25^{\circ} \mathrm{C}(\mathrm{pH} \sim 5.0)\right)$ shows a shear thinning property up to a shear rate of $25 \mathrm{~s}^{-1}$ and then the shear thickening phenomenon starts to occur at low concentrations $(\sim 2 \mathrm{mM})$, but above a shear rate of 60, the fluid shows a Newtonian type behavior [17]. However, an overall non-Newtonian nature is apparent as the concentration of the CTAB and naphthol (1:1) system is raised above $2.0 \mathrm{mM}$. At still higher concentrations $(\sim 5.0 \mathrm{mM})$, the nature of the rheological response 
changes dramatically and the system starts displaying an unusual rheology as a function of shear rate. Up to a shear rate of $60 \mathrm{~s}^{-1}$, the fluid shear thins. An onset of viscosity rise is observed at the shear rate of 60 $\mathrm{s}^{-1}$, and the system again shear thins, passing through a maximum at $70 \mathrm{~s}^{-1}$.

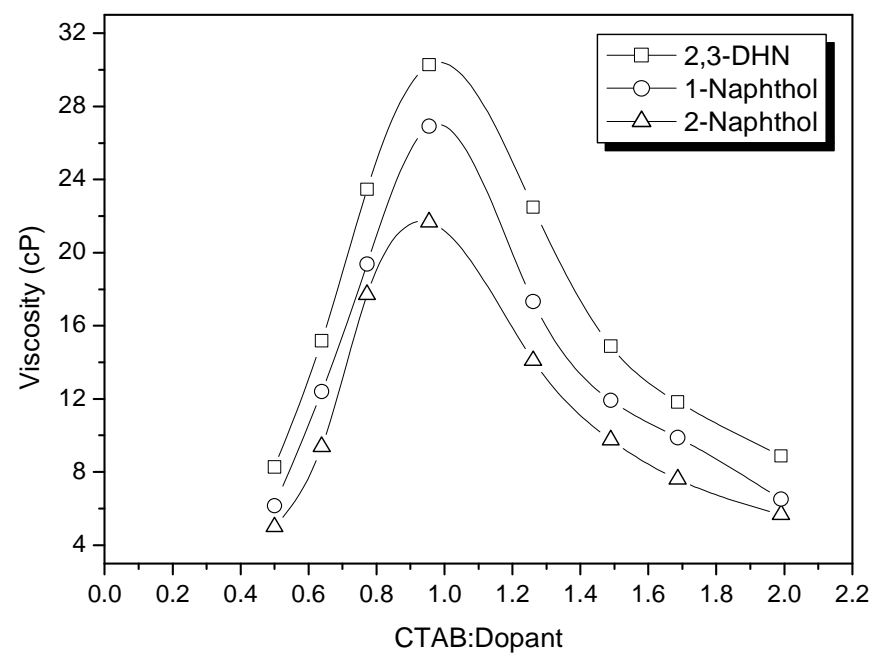

Figure 1: Variation of viscosity as a function mole ratio of CTAB and the dopants (2-Naphthol, 1-naphthol and 2,3-dihydroxynaphthelene) that form viscoelastic gels.

At further higher concentrations $(8.5 \mathrm{mM})$, the viscosity-shear rate profile again changes feature; the initial shear thinning characteristics disappear. The overall behavior is consistent with building up of long worm-like micellar bundles at relatively high concentrations.

Spectral Modification of Micelle-Embedded Dopants: In view of the differences in the viscoelastic responses and the morphological transitions of CTAB micelles induced by neutral naphthols and the methoxynaphthalenes, UV absorption spectra of these dopants may be interesting to study in micellar media. To understand the kind of interactions which are operative in the micelle-dopant systems, the key element of the present study is to compare the spectral characteristics of naphthols (HNs which contain $\mathrm{OH}$ ) with those of methoxy naphthalenes (MNs, which do not contain $\mathrm{OH}$ ) under various conditions in order to visualize a consistent molecular picture eliminating the untenable suggestions. Aromatic compounds, e.g., naphthalene, in general, have two strongly overlapped bands in the near UV region, viz., the longitudinally polarized ${ }^{1} \mathrm{~L}_{\mathrm{a}} \leftarrow{ }^{1} \mathrm{~A}$ band and the transversely polarized ${ }^{1} \mathrm{~L}_{\mathrm{b}} \leftarrow{ }^{1} \mathrm{~A}$ band. While the vibrational structure of these bands appears differently in different substituted compounds, effects of extending conjugation in 1 and 2 positions by $\mathrm{OH}$ or $\mathrm{CH}_{3} \mathrm{O}$ groups in naphthol and methoxynaphthalene molecules, respectively, are interesting. Both in 1-naphthol and 1-methoxy, naphthalene conjugation is extended in the transverse direction and, therefore, it affects the transverse polarized ${ }^{1} \mathrm{~L}_{\mathrm{a}}$ band. In 2-naphthol and 2-methoxy naphthalene, on the other hand, conjugation is primarily extended in the longitudinal direction, affecting both the intensity and the frequency of the longitudinally polarized ${ }^{1} \mathrm{~L}_{\mathrm{b}}$ band compared to the unsubstituted naphthalene.

It is well-known that the near UV spectra of aromatic compounds are affected by specific interactions like hydrogen bonding. Noncovalent interactions like $\pi-\pi$ and cation- $\pi$ also cause shifts in the electron distributions of the molecule. The near UV absorption of 1-naphthol which arises from two strongly overlapped $\pi-\pi^{*}$ transitions remains unaffected in the presence of submicellar aqueous CTAB solution, indicating the absence of any appreciable interaction. The effect of CTAB micelles on the UV spectra of 
2-naphthol is also similar (Fig. 2). However, interestingly, significant red-shift starts to occur $(6.4 \mathrm{~nm}$ at $\left.\lambda_{\max } \sim 293 \mathrm{~nm}\right)$ in the presence of CTAB just above its $\mathrm{cmc}(0.96 \mathrm{mM})$ with a well-defined isobastic point at $296 \mathrm{~nm}$. Such shifting of $\lambda_{\max }$ continues until most of the naphthol molecules are partitioned in the micellar phase at high surfactant/ naphthol ratio (80:1).

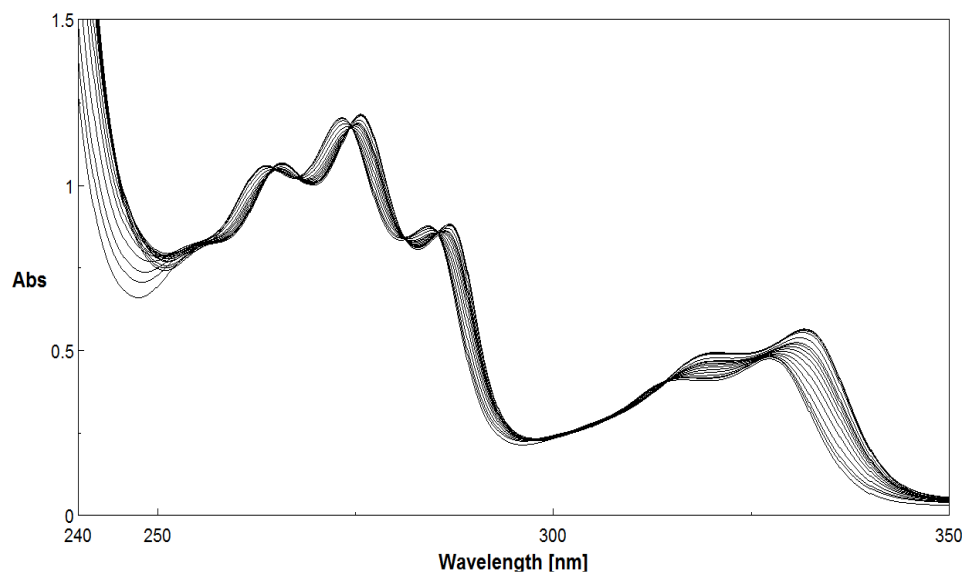

Figure 2: Absorption spectra of 2-Naphthol $(0.25 \mathrm{mM})$ in water at varying concentrations of CTAB at $25{ }^{\circ} \mathrm{C}$. [CTAB]: (1) 0.0, (2) 0.65, (3) 0.79, (4) 0.94, (5) 1.13, (6) 1.36, (7) 1.63, (8) 1.96, (9) 2.35, (10) 2.82,

(11) 3.39, , (12) 5.08, , (13) 7.63, (14) 11.44, (15) 17.16, (16) $20.60 \mathrm{mM}$

The result suggests that the protruded $\mathrm{OH}$ groups of micelle-embedded naphthols form a H-bond with interfacially located $\left(D_{\text {eff }} \sim 45\right)$ water molecules and act as a H-donor. It may also be argued that at a mole ratio of 1:1 of naphthol and the $\mathrm{CTAB}$, at which maximum viscoelastic response is observed under shear due to the presence of entangled worm-like micelles, not all of the naphthol molecules are embedded in the micelles, but some are located in the palisade layer. These naphthols may, however, be involved in $\mathrm{H}$ bond network formation with embedded molecules via interfacial water. The relatively less polar and less mobile water molecules compared to bulk water form a strong $\mathrm{H}$-bond with the $\mathrm{OH}$ group of embedded naphthols, which act as H-donors and result in an optimum orientation of aromatic $\pi$-electron systems in the micelles to shield the surfactant headgroup charges efficiently; may be via cation- $\pi$ interaction; i.e., the cation charge of surfactant head groups interacts with the quadrupole moment of the aromatic $\pi$ system of naphthols. On the other hand, as the $\mathrm{H}$ atom of $\mathrm{OH}$ is replaced by a $\mathrm{CH}_{3}$ group (viz., the methoxynaphthalene molecule), the ability of intermolecular H-bond formation disappears. Instead, the $\mathrm{H}$-accepting tendency from a potential donor is enhanced. The nature of changes encountered in the UV spectra of methoxynaphthalenes on the addition of CTAB above its cmc indicates the permeation of the dopant molecules in the micelles. The small red-shift compared to that in naphthols indicates a weaker noncovalent interaction takes place. The large drop in intensity on first addition of $0.33 \mathrm{mM} \mathrm{CTAB}$ is the signature of breaking of a H-bond with bulk water molecules. Due to their directionality and spatial arrangement, complementary multiple $\mathrm{H}$-bonding interactions at the micellar interface lead to engineering well-defined supramolecular structure via micellar headgroup charge shielding by $\pi$-electron systems of naphthols.

Shear-Induced Viscosity and pH: The role of neutral hydroxyaromatic dopants, viz., 1- and 2naphthol, stimulates the idea of designing a route for $\mathrm{pH}$-responsive vesicle formation. This idea stems from the fact that the dopants, which under neutral conditions activate the formation of worm-like micelles at $\mathrm{pH} \sim 5.0$, may on partial ionization of the $\mathrm{OH}$ group increase the packing parameter further via charge screening. While the viscosity of both, 2- as well as 1-HN-CTAB systems, is quite high due to 
formation of long worm-like micelles at low $\mathrm{pH}$, the viscosity of the former system falls initially, indicating formation of shorter micelles with $\mathrm{pH}$ until $\mathrm{pH} \sim 5.0$ is reached. On the other hand, for 1-HN$\mathrm{CTAB}$, the onset of viscosity rise as a function of $\mathrm{pH}$ is found to occur from very low $\mathrm{pH}(\mathrm{pH} \sim 2.0)$. For 2-naphthol-CTAB, the onset of viscosity rise is observed at higher $\mathrm{pH}(\sim 5.0)$ and the viscosity-pH profile passes through a maximum at $\mathrm{pH} \sim 8.5$. Further increase in $\mathrm{pH}$ results in the ionization of $\mathrm{OH}$ group further, and the packing parameter probably exceeds the critical value of $1 / 2$ via enhanced charge screening, leading to vesicle formation (for naphthols, $\mathrm{pKa} \sim 9$, which means $50 \%$ ionization of the $\mathrm{OH}$ group at $\mathrm{pH} \sim 9.0$ ). This results in the fall of viscosity of the system. Since 1-naphthol could modulate the micellar surface curvature more efficiently, a little dissociation of the $\mathrm{OH}$ group (at low $\mathrm{pH}$ range) leads to an appreciable decrease of surface curvature via charge screening and promotes long worm-like micelle formation. In fact, for the 1-HN-CTAB system, vesicles start to form even at slightly higher than $\mathrm{pH} \sim 5.0$. A simple and effective route to design $\mathrm{pH}$ responsive viscoelastic worm-like micelles and less viscous globular vesicles based on naphthol dopants may be tuned by controlling the degree of charge screening of $\mathrm{CTAB}$ micelles via controlled ionization of naphtholic $\mathrm{OH}$ groups. The result of $\mathrm{pH}$-responsive morphology modification is further investigated by means of cryo-TEM.

Cryogenic Transmission Electron Microscopic Study: Cryo-TEM images of the CTAB-2naphthol system at high $\mathrm{pH}$ 's is shown in Fig. 3. At high $\mathrm{pH}(\mathrm{pH} 9.4)$, the system contains very long wormlike micelles, which coexist with large unilamellar vesicles. This is undoubtedly due to enhanced charge screening of micelles by naphtholate anions. The field is seen to populate mainly by large vesicles of diameter $\sim 30 \mathrm{~nm}$ along with thinly populated smaller vesicles. It is also seen that the long worm-like micelles are highly entangled. Sometimes they are found to elongate linearly under shear flow. The solutions are completely transparent. The direct imaging by cryo-TEM supports the rheological observation as a function of $\mathrm{pH}$. At low $\mathrm{pH}$, the worm-like micelles are formed via headgroup charge shielding by aromatic $\pi$ electrons, whereas, at high $\mathrm{pH}$, ionization of $\mathrm{OH}$ groups takes place and the packing parameter exceeds the critical value of $1 / 2$ via enhanced charge screening by naphtholate ion. This leads to unilamellar vesicle formation along with long worm-like micelles.

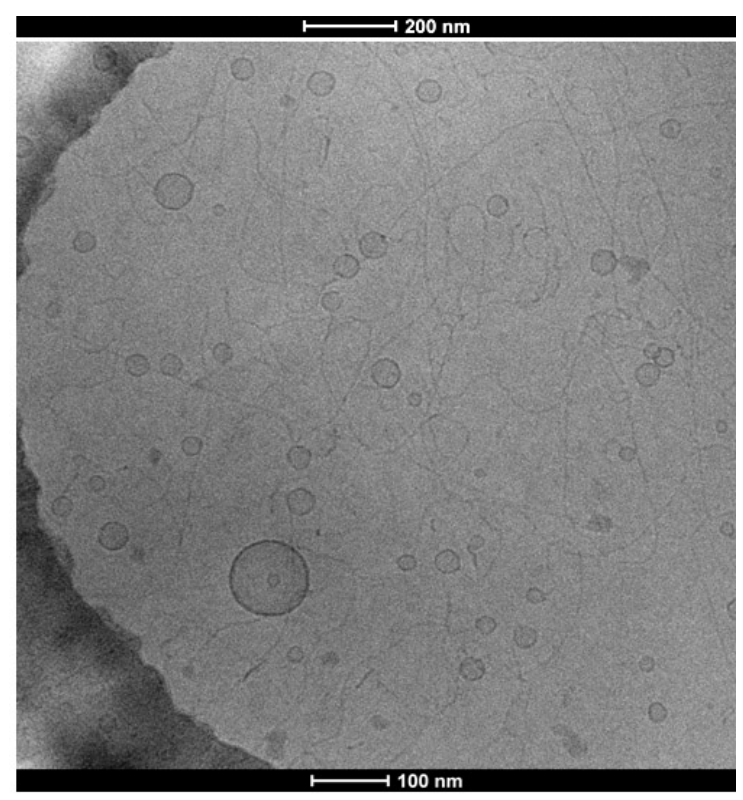

Figure 3: Cryo-TEM image of CTAB-2-naphthol at a pH of 9.4. [15] 


\section{References}

[1] J.M. Lehn, Science, 295 (2002) 2400.

[2] G. M. Whitesides, M. Boncheva, Proc. Natl. Acad. Sci. U. S. A., 99 (2002) 4769.

[3] J. C. Hao, H. Hoffmann, Curr. Opin. Colloid Interface Sci., 9 (2004) 279.

[4] S. J. Candau, E. Hirsch, R. Zana, J. Colloid Interface Sci., 105 (1985) 521.

[5] H. Rehage, H. Hoffmann, Mol. Phys., 74 (1991) 933.

[6] S. Ezrahi, E. Tuval, A. Aserin, Adv. Colloid Interface Sci., 128 (2006) 77.

[7] L. M. Walker, Curr. Opin. Colloid Interface Sci., 6 (2001) 451.

[8] G. C. Maitland, Curr. Opin. Colloid Interface Sci., 5 (2000) 301.

[9] H. Rehage, H. Hoffmann, J. Phys. Chem., 92 (1988) 4712.

[10] P. A. Hassan, S. R. Raghavan, E. W. Kaler, Langmuir, 18 (2002) 2543.

[11] S. Imai, T. Shikata, J. Colloid Interface Sci., 244 (2001) 399.

[12] K. Bijma, J. B. F. N. Engberts, Langmuir, 13 (1997) 4843.

[13] R. Abdel-Rahem, M. Gradzielski, H. Hoffmann, J. Colloid Interface Sci., 288 (2005) 570.

[14] S. K. Saha, M. Jha, M. Ali, A. Chakraborty, G. Bit, S.K. Das, J. Phys. Chem. B, 112 (2008) 4642.

[15] M. Ali, M. Jha, S. K. Das, S. K. Saha, J. Phys. Chem. B, 113 (2009) 15563.

[16] M. Ali, S.K. Saha, Hydrogen Bonding and Transfer in the Excited State, Volume I \& II (eds K.-L. Han and G.-J. Zhao), John Wiley \& Sons, Ltd., Chichester, UK, 2010.

[17] G. Tan, C. Ford, V. T. John, J. He, G.L. McPherson, A. Bose, Langmuir, 24 (2008) 1031.

[18] M. Isayama, K. Nomiyama, T. Yamaguchi, N. Kimizuka, Chem. Lett., 34 (2005) 462.

[19] M. Cates, S.J. Cardau, J. Phys., 2 (1990) 6869.

[20] F. Kern, R. Zane, S.J. Caddau, Langmuir, 7 (1991) 1344.

[21] Z. Liu, J. Cai, L.E. Seriven, H.T Davis, J. Phys. Chem., 98 (1994) 5984.

[22] P. Boltenhagen, Y. Hu, E.F Matthys, D.J.Pine, Phys. Rev. Letts., 79 (1997) 2359.

[23] S.L. Keller, P. Boltenhagen, D.J. Pine, J.A. Zasadzinski, Phy. Rev. Letts., 12 (1998) 2725.

[24] R.D. Koehler, S.R.Raghavan, E.W. Kaler, J. Phys. Chem. B, 104 (2000) 11035.

[25] T. Emae, Colloid. Polym. Sci., 267 (1989) 707.

[26] T. Emae, S. Ikeda, J. Phy. Chem., 96 (1986) 5216.

[27] G.C. Kalur, S.R. Raghavan, J. Phys. Chem., B 109 (2005) 8599.

[28] V. Agarwal, M. Singh, G. Mc. Pherson, V.T. John, A. Bose, Colloid. Surf. A, 281 (2006) 246.

[29] M. Isayama, K. Nomiyama, T.Yamaguchi, N. Kimizuka, Chem. Letts., 34 (2005) 462.

[30] R.G. Shrestha, K. Sakai, H. Sakai, M. Abe, J. Phys. Chem. B, 115 (2011) 2937.

[31] W.C. Zhang, G.Z. Li, Q. Shen, J.H. Mu, Colloids. Surf. A, 170 (2000) 59.

[32] Z. Zhu, Y.I. Gonzalez, H. Xu, E.W. Kaler, S. Liu, Langmuir, 22 (2006) 949.

[33] Y. Liu, Xue Han, J. Huang, H. Fu, C. Yu, J. Colloid Interface Sci., 330 (2009) 449.

[34] Dong, J. Zhang, L. Zheng, S. Wang, X. Li, T. Inoue, J. Colloid Interface Sci., 319 (2008) 338. 